The first galaxies in the very early universe 


\title{
Simulating the First Galaxies
}

\author{
Volker Bromm \\ Department of Astronomy, University of Texas, \\ 2511 Speedway, RLM 13.116, Austin, TX 78712, U.S.A. \\ email: vbromm@astro.as.utexas.edu
}

\begin{abstract}
The formation of the first galaxies marks the end of the cosmic dark ages, initiating the prolonged process of reionization and enriching the pristine intergalactic medium with the first heavy chemical elements. It is now possible to simulate this process with ever greater detail of physical realism, while still considering the proper cosmological context. The simulations have taught us that the feedback from Population III stars is vital in shaping the properties of early galaxies. We are close in pushing ab initio simulations to the point where future instruments, such as the James Webb Space Telescope, can directly test theoretical predictions.
\end{abstract}

Keywords. cosmology: theory, early universe, galaxies: formation, stars: formation

\section{Introduction}

The first sources of light fundamentally transformed the early universe, from the simple initial state of the cosmic dark ages into one of proliferating complexity (Barkana \& Loeb 2001; Bromm et al. 2009; Loeb 2010). This process began with the formation of the first stars, the so-called Population III (Pop III), at redshifts $z \sim 20-30$. These stars are predicted to form in dark matter minihalos, comprising total masses of $\sim 10^{6} M_{\odot}$. Current models suggest that Pop III stars were typically massive, or even very massive, with $M_{*} \sim 10-100 M_{\odot}$; these models also predict that the first stars formed in small groups, including binaries or higher-order multiples. These developments are further discussed below.

Once the first stars had formed, feedback processes began to modify the surrounding intergalactic medium (IGM). It is useful to classify them into 3 categories (Ciardi \& Ferrara 2005): radiative, mechanical, and chemical. The first feedback consists of the hydrogen-ionizing photons emitted by Pop III stars, as well as the less energetic, moleculedissociating radiation in the Lyman-Werner (LW) bands. When the first stars die, after their short life of a few million years, they will explode as a supernova (SN), or directly collapse into massive black holes. In the SN case, mechanical and chemical feedback come into play. The SN blastwave exerts a direct, possibly very disruptive, effect on its host system, whereas the chemical feedback acts in a more indirect way, as follows: The first stars, forming out of metal-free, primordial gas, are predicted to be characterized by a top-heavy initial mass function (IMF). Once the gas had been enriched to a threshold level, termed "critical metallicity" $\left(Z_{\text {crit }}\right)$, the mode of star formation would revert to a more normal IMF, which is dominated by lower mass stars (Frebel et al. 2007). Chemical feedback refers to this transition in star formation mode, implying that less massive stars have a less disruptive impact on their surroundings. Pre-galactic metal enrichment, the transport and mixing of the first heavy elements into the pristine IGM, thus governs the Pop III - Pop II transition (Tornatore et al. 2007; Maio et al. 2010). Although crucially important, this early enrichment episode is still poorly understood (reviewed in Karlsson et al. 2012). 
To gauge the strength of the feedback exerted by Pop III stars, the key is to consider that their formation sites, the minihalos mentioned above, have shallow gravitational potential wells. The corresponding virial temperatures, $T_{\text {vir }} \sim 10^{3} \mathrm{~K}$, indicate that such halos cannot confine photoheated gas. As Pop III stars were typically massive, they would quickly exert a strong negative feedback on their host systems. Numerical simulations indicate that in extreme cases this feedback completely destroys the host, in the sense of heating and evacuating all remaining gas. There would therefore be no opportunity for a second burst of star formation in a minihalo. In the case of a less top-heavy IMF, the initial negative feedback would still be strong, but the recovery timescale for enabling second-generation star formation could be significantly reduced. Since all (most?) Pop III stars are massive enough to quickly die, there would be no long-lived system of low-mass stars left behind. The Pop III forming minihalos, therefore, are not galaxies, if a bona-fide galaxy is meant to imply a long-lived stellar system, embedded in a dark matter halo. The question: What is a galaxy, and, more specifically, what is a first galaxy?, however, is a matter of ongoing debate (Bromm et al. 2009; Forbes \& Kroupa 2011; Willman \& Strader 2012). As we have seen, this question is intimately tied up with the feedback from the first stars, which in turn is governed by the Pop III IMF (top-heavy or more normal).

Theorists are currently exploring the hypothesis that "atomic cooling halos" are viable hosts for the true first galaxies (Oh \& Haiman 2002). These halos have deeper potential wells, compared to minihalos, characterized by virial temperatures of $T_{\text {vir }} \simeq 10^{4} \mathrm{~K}$, enabling the primordial gas to cool via efficient line emission from atomic hydrogen. It is useful to keep in mind that observers and theorists often employ different definitions for 'first galaxy'. As a theorist, you wish to identify the first, i.e., lowest-mass, dark matter halos that satisfy the conditions for a galaxy. Observers, on the other hand, usually aim at detecting truly metal-free, primordial systems. Recent simulation results, however, suggest that such metal-free galaxies do not exist. The reason being that rapid SN enrichment from Pop III stars, formed in the galaxy's minihalo progenitors, provided a bedrock of heavy elements (Greif et al. 2010; Bromm \& Yoshida 2011; Wise et al. 2012). Any second generation stars would then already belong to Population II (Pop II).

The first star and galaxy field is just entering a dynamic phase of rapid discovery. This development is primarily driven by new technology, on the theory side by ever more powerful supercomputers, reaching peta-scale machines, and on the observational side by next-generation telescopes and facilities. Among them are the James Webb Space Telescope (JWST), planned for launch in 2018 (Gardner et al. 2006), and the suite of extremely large, ground-based telescopes, such as the GMT, TMT, and E-ELT. Complementary to them are ongoing and future meter-wavelength radio arrays, designed to detect the redshifted $21 \mathrm{~cm}$ radiation from the neutral hydrogen in the early universe (Furlanetto et al. 2006). A further intriguing window into the epoch of the first stars is provided by high-redshift gamma-ray bursts (GRBs). These are extremely bright, relativistic explosions, triggered when a rapidly rotating massive star is collapsing into a black hole (Bloom 2011). The first stars are promising GRB progenitors, thus possibly enabling what has been termed "GRB cosmology" (Lamb \& Reichart 2000; Bromm \& Loeb 2006). Of great promise is to use such high- $z$ bursts as bright background sources to probe the ionization and metal enrichment state of the early IGM (Wang et al. 2012).

There is a second approach to study the ancient past, nicely complementary to the in situ observation of high-redshift sources. This alternative channel, often termed "NearField Cosmology" (Freeman \& Bland-Hawthorn 2002), is provided by local fossils that have survived since early cosmic times. Among them are extremely metal-poor stars found in the halo of the Milky Way. The idea here is to scrutinize their chemical abundance 
patterns and derive constraints on the properties of the first SNe, and, indirectly, of the Pop III progenitor stars, such as their mass and rate of rotation (Beers \& Christlieb 2005; Frebel 2010; Tumlinson 2010). Another class of relic objects is made up of the newly discovered extremely faint dwarf galaxies in the Local Group. These ultra-faint dwarf (UFD) galaxies consist of only a few hundred stars, and reside in very low-mass dark matter halos. Their chemical and structural history is therefore much simpler than what is encountered in massive, mature galaxies, and it should be much more straightforward to make the connection with the primordial building blocks (Salvadori \& Ferrara 2009; Bovill \& Ricotti 2011a, Bovill \& Ricotti 2011b; Frebel \& Bromm 2012).

In the following, we will briefly review some of the key physical ingedients involved in simulating the formation of primordial galaxies (for further details, see Bromm \& Yoshida 2011).

\section{Feedback from the first stars}

The assembly process of the first galaxies sensitively depends on the feedback exerted by Pop III stars formed in the progenitor minihalos. Different assumptions on the primordial IMF will thus lead to divergent first galaxy formation histories and resulting properties (Pawlik et al. 2011, Pawlik et al. 2013). Here, we will comment on some issues that are vigorously debated, without attempting a complete exposition of the vast subject of feedback (for further discussion, see Ciardi \& Ferrara 2005). In this context, it is important to keep in mind two related points. First, even a single Pop III star can exert an impact on a cosmological scale, possibly affecting the entire Lagrangian volume that is destined to collapse into the first galaxy later on. Second, the number of possible progenitor stars is quite limited (Wise \& Abel 2007; Greif et al. 2008). The merger tree of an atomic cooling halo contains of order 10 minihalos. If one then assumes that only a small multiple of Pop III stars forms per minihalo, as suggested by recent simulations, one has the same order of progenitor Pop III stars. The Pop III feedback events will thus sample a possibly broad primordial IMF, thus likely introducing a range in first star properties, such as stellar mass, average metallicity, and luminosity.

\subsection{Population III Mass Scale}

The longstanding consensus view has been that the conditions in the early universe favored the formation of predominantly massive stars, such that the Pop III IMF was top-heavy (Abel et al. 2002; Bromm et al. 2002; Bromm \& Larson 2004; Glover 2005). This expectation rests on the much less efficient cooling in pure $\mathrm{H} / \mathrm{He}$ gas, where the only viable cooling agent is molecular hydrogen. The primordial gas can then cool to only about $\sim 200 \mathrm{~K}$, compared to the $10 \mathrm{~K}$ reached in dust-cooled molecular clouds in the present-day Milky Way. The correspondingly enhanced thermal pressure is reflected in a Jeans mass that is larger by one to two orders of magnitude in the Pop III case. If cooling due to hydrogen deuteride, $\mathrm{HD}$, were to become important, the primordial gas could reach somewhat lower temperatures, possibly down to the temperature of the cosmic microwave background (Johnson \& Bromm 2006). The Jeans mass, and therefore the scale of fragmentation, may then also have been somewhat lower, giving rise to what has been termed 'Pop III.2' stars (McKee \& Tan 2008). Another element of this 'standard model' of primordial star formation has been that the first stars formed typically in isolation, one per minihalo.

Recently, beginning with work done in 2009, this traditional paradigm has been refined in important ways (Turk et al. 2009; Stacy et al. 2010; Clark et al. 2011b; Greif et al. 2011, Greif et al. 2012). Supercomputing power, as well as algorithmic advances, now 
enable us to follow the protostellar collapse to densities where the initial hydrostatic core forms in the center of the cloud, at $n \sim 10^{22} \mathrm{~cm}^{-3}$ (Yoshida et al. 2006, Yoshida et al. 2008), and, crucially, beyond this stage into the main accretion phase. These simulations have demonstrated that accretion is mediated through a near-Keplerian disk. The hot conditions in the surrounding cloud result in extremely large rates of infall onto the disk $\left(\dot{M} \propto T^{3 / 2}\right)$; this rapid mass loading drives the disk inevitably towards gravitational instability, such that a small multiple of Pop III protostars emerges, often dominated by a binary system (see Fig. 1). It is not yet possible to push such ab initio simulations all the way to the completion of the protostellar assembly process; the final mass of Pop III stars and their final IMF are thus still subject to considerable uncertainty.

However, first attempts to carry out the radiation-hydrodynamical calculations required to treat the late accretion phase, where protostellar feedback tends to limit further infall, have confirmed the basic prediction: the first stars were typically massive, with masses of a few $\sim 10 M_{\odot}$, although rarely very massive $\left(>100 M_{\odot}\right)$, as previously thought (McKee \& Tan 2008; Hosokawa et al. 2011; Stacy et al. 2012). More specifically, the radiative feedback operates through the agency of an ultra-compact H II region, expanding perpendicularly to the disk, once the first protostar has reached a mass, $M_{*} \gtrsim 10 M_{\odot}$, and surface temperature that is sufficiently hot to produce ionizing photons. This hourglass-shaped ionized region then acts back on the dense, neutral gas in the disk, slowly photo-evaporating it (McKee \& Tan 2008). With the reservoir for further growth gone, the Pop III star has reached its final mass. This process will depend on the rotation state of the accreting protostar (Stacy et al. 2013), because different rotation speeds lead to different protostellar radii and therefore effective temperatures. There is a similar dependence of radius on the rate of accretion (Smith et al. 2012). The upper mass limit, set via radiative feedback, will thus exhibit a possibly wide range.

\subsection{Supernova Feedback}

Supernova feedback impacts first galaxy formation in multiple ways (Salvaterra et al. 2011). The related energy input tends to partially disrupt the minihalo host, delaying any second-generation star formation. Within the previous paradigm of extremely massive Pop III stars, attention has been focused on pair-instability supernovae (PISNe), with explosion energies that can be larger than for conventional core-collapse events by up to two orders of magnitude (Hummel et al. 2012; Pan et al. 2012). In this case, the negative feedback is severe, and next-generation star formation is delayed by a considerable fraction of the local Hubble time (Wise \& Abel 2008; Greif et al. 2010). Indeed, the severity of such energetic SN feedback is largely responsible for shifting the mass scale of first galaxy, and therefore second-generation star formation, hosts to $\sim 10^{8} M_{\odot}$ (atomic cooling halos). If, on the other hand, one considers Pop III progenitor masses that are less extreme, in line with the most recent findings, any feedback effects will be less disruptive, and recovery timescales to enable next-generation star formation could be less, typically a few million years (see Fig. 2). As a corrolary, one then also gets less massive host systems for the first galaxies, possibly below the threshold for atomic hydrogen cooling (Ricotti et al. 2002a, Ricotti et al. 2002b). One should keep in mind, however, that extremely energetic explosions may be triggered even in the death of progenitor stars of more modest mass, provided that they are rapidly rotating. Recent simulations have indeed found hints for such high-spin conditions (Stacy et al. 2011, Stacy et al. 2013), possibly giving rise to hypernova explosions which would have a very similar effect on first galaxy assembly as a PISN (Greif et al. 2010).

Next, the Pop III SNe disperse heavy-elements into the surrounding medium, thus fundamentally changing the conditions for second-generation star formation. Once 

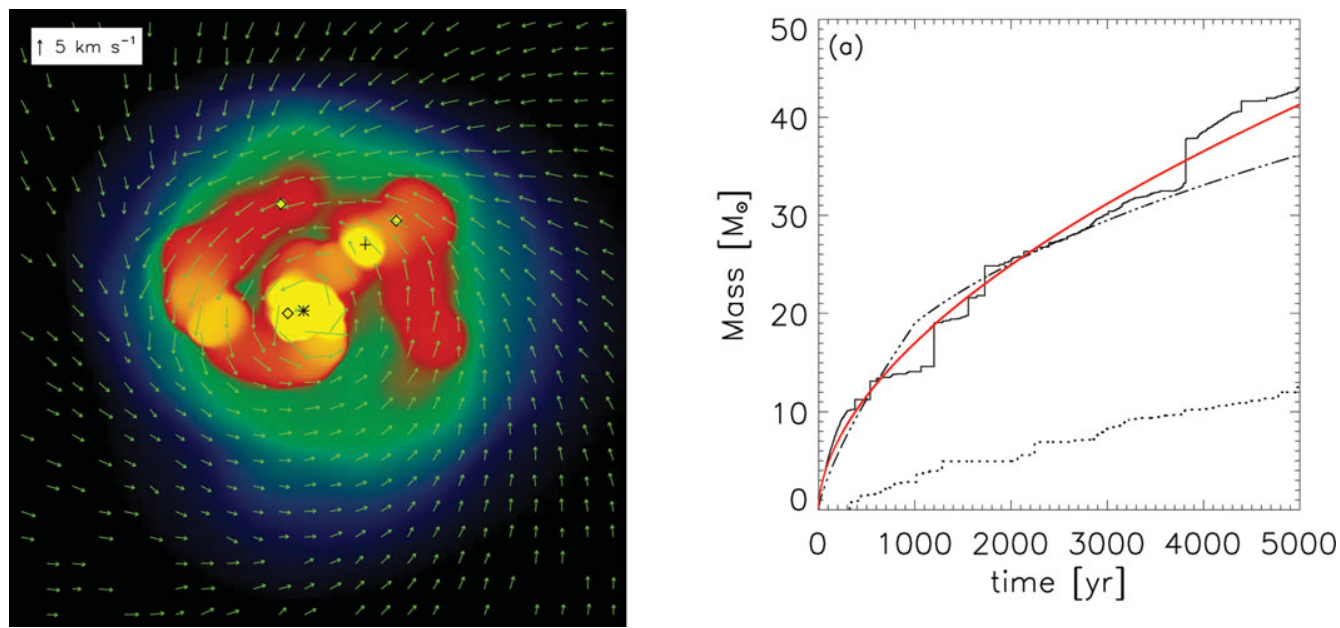

Figure 1. Simulating Pop III star formation (from Stacy et al. 2010). Left panel: Multiple protostars embedded in an accretion disk. The colors symbolize the underlying density field (yellow marks highest density) within the central 5000 AU. Here, protostars are represented by sink particles, such that the asterisk marks the location of the most massive sink, the cross that of the second most massive one, and diamonds represent the other, smaller sinks. Shown is the situation $5000 \mathrm{yr}$ after initial sink formation. At this point, an ordered, nearly Keplerian velocity structure has been established within the disk. Right panel: Sink mass vs. time. The solid line shows the mass of the first sink particle, fitted by a power law according to $M \propto t^{0.55}$ (red line). The dash-dotted line depicts the growth law found in an earlier, lower-resolution simulation (Bromm \& Loeb 2004). The dotted line traces the mass growth of the second largest sink. As can be seen, sinks grow to masses $>10 M_{\odot}$ within a few $1,000 \mathrm{yr}$.

metallicity levels exceed the critical value, $Z_{\text {crit }}$, predicted by theory, stars will form with a more normal IMF, giving rise to the first low-mass (Pop II) stars. The detailed physics of this Pop III to II transition is complex; one issue being whether cooling from fine-structure lines dominates (Bromm \& Loeb 2003a), or that from dust grains (Schneider et al. 2006). As even a single Pop III SN can already enrich the gas in the center of a first galaxy to levels of $Z>10^{-3} Z_{\odot}$, larger than any of the cooling thresholds (dust or other), one gets the robust prediction: The majority of the first galaxies were already metal-enriched, and thus hosted long-lived Pop II stars (Johnson et al. 2008; Wise \& Abel 2008; Greif et al. 2010; Wise et al. 2012). The presence of heavy-element coolants may also be able to significantly boost star formation efficiencies. Simulations tell us that these are rather low in primordial gas: $\eta_{*}=M_{*} / M_{\text {gas }} \sim 10^{-3}$, where $M_{\text {gas }}$ is the total baryonic (=gas) mass in a given host system (here a minihalo). The same inefficiency may also apply to primordial star formation in more massive host systems (Safranek-Shrader et al. 2012). A metal-induced boost in $\eta_{*}$ is thus essential to form massive stellar (Pop II) clusters. It is an interesting problem for 'stellar archaeologists' to (spectroscopically) identify those fossil Pop II stars in our Milky Way and Local Group (see the references given above), providing us with a powerful probe of the conditions in the first galaxies.

\subsection{Black Hole Feedback}

For a range of Pop III progenitor masses, the star is predicted to collapse, directly or in a delayed fashion, into a black hole $(\mathrm{BH})$. This opens up the possibility for a qualitatively different class of feedback effects. Accretion onto the $\mathrm{BH}$, either from the surrounding diffuse medium or a less-massive companion in a binary (HMXB) system, will 


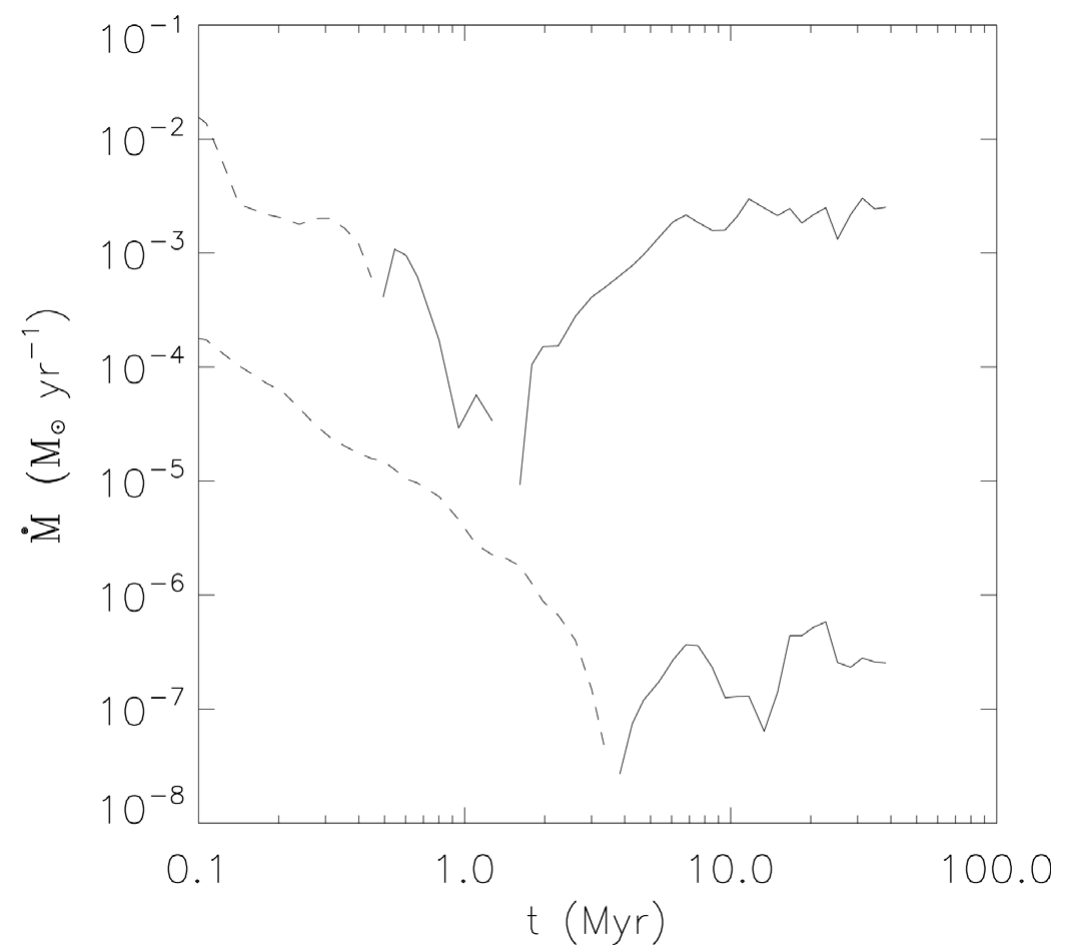

Figure 2. Pop III Supernova feedback (from Ritter et al. 2012). Here, the simulation traces the case of a less energetic explosion $\left(E_{\mathrm{SN}}=10^{51} \mathrm{ergs}\right)$, corresponding to a progenitor star of $\sim 40 M_{\odot}$. Metal (lower curve) and total baryon (upper curve) net mass flow through a sphere of radius $20 \mathrm{pc}$, centered on the gravitational potential minimum. Dashed lines indicate outflows and solid lines inflows. Net outflow reverses into an inflow earlier in the baryons because of the presence of cold filaments delivering metal-free gas from the cosmic web into the halo center.

lead to non-thermal X-ray emission. Accretion is typically weak in the former case, where Bondi-Hoyle accretion occurs, and the resulting emission luminosities are strongly subEddington (Milosavljević et al. 2009a, Milosavljević et al. 2009b). Even then, however, the corresponding photoionization heating has a strong negative effect locally, but virtually none on larger scales (see Fig. 3). In the case of a HMXB source, on the other hand, where X-ray fluxes are typically very high for a limited time, there is a global impact on star formation (Mirabel et al. 2011); now, the strong X-ray flux can partially ionize the IGM out to large distances, including the gas in neighboring minihalos. The boost in the free-electron fraction in turn catalyzes the increased formation of $\mathrm{H}_{2}$ molecules, such that cooling, and therefore Pop III star formation, is enhanced as well. Consequently, the global feedback here is positive, whereas locally the effect is again negative (Jeon et al. 2012).

A related problem is whether Pop III BH seeds may be able to grow into supermassive $\mathrm{BHs}$ that can power the high-redshift quasars already found at $z \sim 6-7$. The answer is to the affirmative, provided that growth can proceed at near-Eddington rates (Loeb 2010). However, strong negative feedback effectively limits growth to small fractions of the Eddington rate, because the photoionization-heating around the $\mathrm{BH}$ progenitor star evaporates the gas from the center, such that the holes find themselves in a virtual vacuum for a substantial part of the local Hubble time (Johnson \& Bromm 2007). The correspondingly low rates of accretion severely challenge any theory that tries to explain the high- $z$ quasar hosts with accretion onto (Pop III) stellar seeds. Alternative, more 


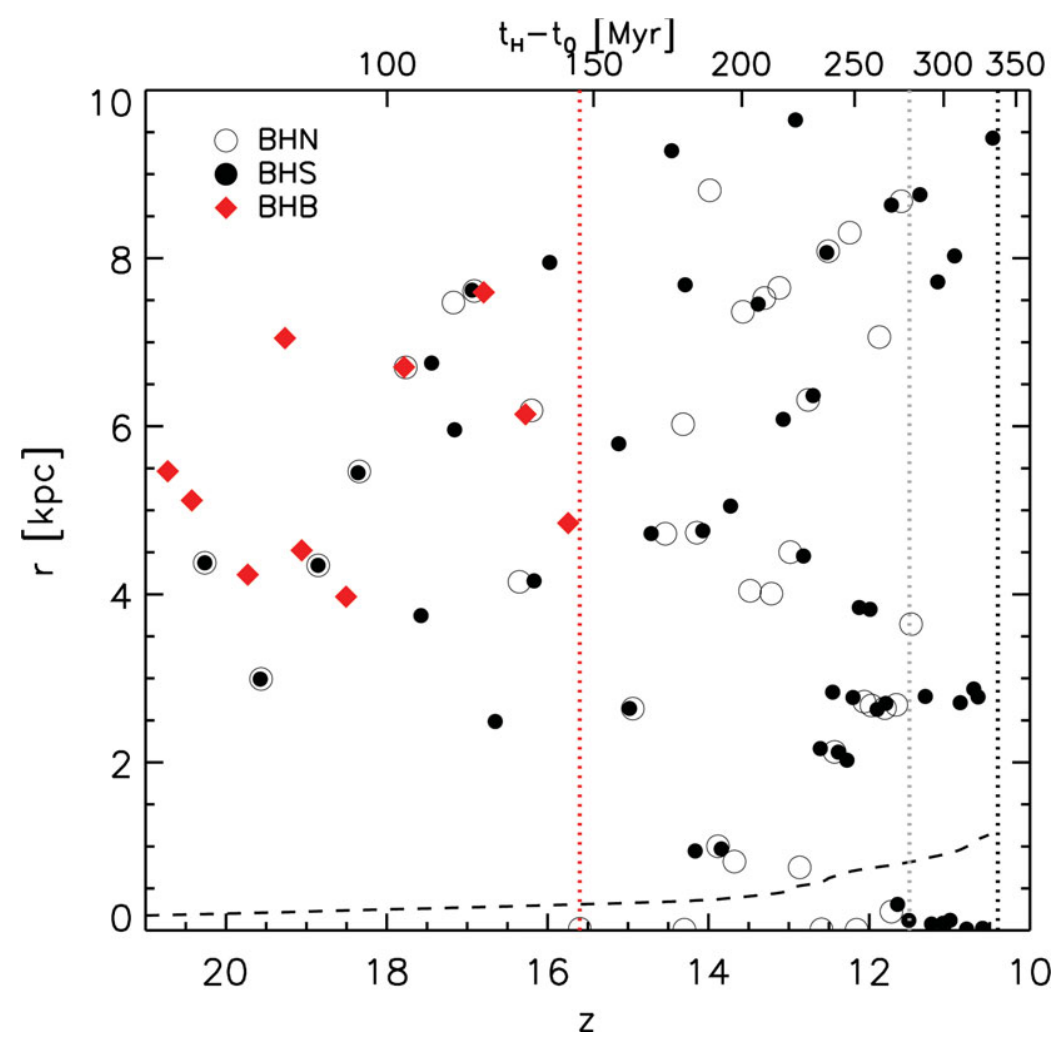

Figure 3. Black hole feedback during first galaxy assembly (from Jeon et al. 2012). Distances between newly formed Pop III stars and the $\mathrm{BH}$ at the center of the emerging first galaxy as a function of redshift. The symbols refer to three related simulations, where the only difference is what is assumed for the BH feedback. Specifically, BHN refers to no BH feedback present, BHS to feedback from a single BH, and BHB to that from a binary (HMXB) source. In the BHB run, positive feedback is evident far away from the source, where gas collapses into distant minihalos, facilitated via $\mathrm{H}_{2}$ cooling promoted by the strong X-ray emission; locally, star formation is suppressed. The virial radius of the halo hosting the active BH or the HMXB is shown for reference (dashed line).

exotic, scenarios have therefore been explored, such as the direct collapse of a primordial gas cloud under strong LW radiation. Molecules may then never form, and consequently no star formation would occur; the cloud may then be able to directly collapse into a (super-) massive BH seed in the absence of any stellar feedback (Bromm \& Loeb 2003b; Volonteri 2012).

\section{Turbulence in the first galaxies}

With the emergence of the first galaxies, we witness the onset of supersonic turbulence, which is expected to have important consequences for star formation (Wise \& Abel 2007; Greif et al. 2010; Prieto et al. 2011). Indeed, the Reynolds number in the center of the first galaxies is very large, $R e \sim 10^{9}$, indicating a highly-turbulent situation, and the Mach number, $M a \sim V / c_{s} \sim v_{\mathrm{vir}} / c_{s} \sim 10$, indicates supersonic flows. In the last estimate, we have used the virial velocity inside an atomic cooling halo, $v_{\mathrm{vir}} \sim 10 \mathrm{~km} \mathrm{~s}^{-1}$, and the sound-speed of $\mathrm{H}_{2}$-cooled gas $\left(c_{s} \sim 1 \mathrm{~km} \mathrm{~s}^{-1}\right)$. In the minihalos, due to their smaller 


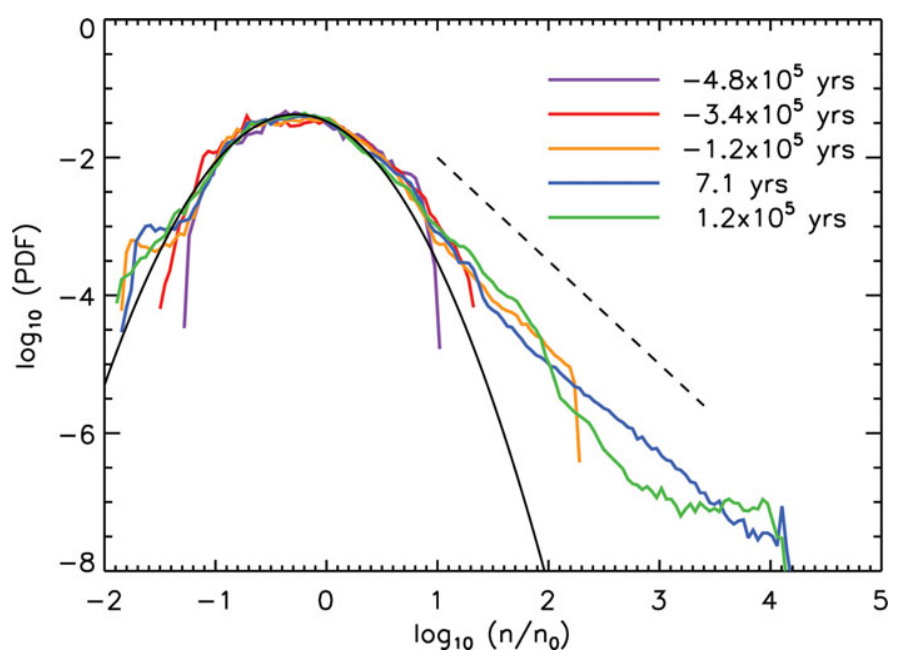

Figure 4. Density structure in turbulent primordial gas (from Safranek-Shrader et al. 2012). The presence of supersonic turbulence is manifested in the characteristic log-normal probability distribution. At late times, the effect of self-gravity imprints a power-law extension towards the highest densities. It is possible that the turbulently structured gas will give rise to a high-mass slope in the stellar IMF similar to the present-day Salpeter one.

virial velocities, which are of order the sound speed, any low-level turbulence is mostly subsonic (Clark et al. 2011a).

Supersonic turbulence generates density fluctuations in the central gas cloud. Statistically, these can be described with a log-normal probability density function (PDF):

$$
f(x) d x=\frac{1}{\sqrt{2 \pi \sigma_{x}^{2}}} \exp \left[-\frac{\left(x-\mu_{x}\right)^{2}}{2 \sigma_{x}^{2}}\right] d x,
$$

where $x \equiv \ln (\rho / \bar{\rho})$, and $\mu_{x}$ and $\sigma_{x}^{2}$ are the mean and dispersion of the distribution, respectively. The latter two are connected: $\mu_{x}=-\sigma_{x}^{2} / 2$ (McKee \& Ostriker 2007). Numerical simulations have shown that the dispersion of the density PDF is connected to the Mach number of the flow: $\sigma_{x}^{2} \simeq \ln \left(1+0.25 M a^{2}\right)$. Inside the first galaxies, one finds values close to $\sigma_{x} \simeq 1$ (Safranek-Shrader et al. 2012). Similar to the well-studied case of isothermal, supersonic turbulence, the central gas in the first galaxies exhibits the imprint of self-gravity (Fig. 4): a power-law tail toward the highest densities, on top of the log-normal PDF at lower densities, which is generated by purely hydrodynamical effects (Safranek-Shrader et al. 2012).

\section{Future prospects}

The coming decade will likely see a flurry of discoveries in the pre-reionization universe, getting us closer to answering some of the questions of the ages: What are our cosmic origins and how did it all begin? Technology, involving both next-generation observational facilities and peta-scale supercomputing, will likely play a prominent role in this endeavor. Thus probing the first galaxies may provide us with an ideal, simplified laboratory for the otherwise exceedingly complex problem of galaxy formation and evolution in general. Simulation-based predictions will be crucial in guiding the design and observing strategies of future telescopes, in effect providing signposts in the dark, at the very frontier of our 
knowledge of the early universe. It is clear that serendipity will be involved, but with a bit of luck, dark-age cosmology should soon come into its own.

\section{Acknowledgments}

I would like to thank the organizers for putting together a stimulating program, providing an exciting new perspective on massive galaxy formation. Support from NSF grant AST-1009928 and NASA ATFP grant NNX09AJ33G is gratefully acknowledged.

\section{References}

Abel, T., Bryan, G. L., \& Norman, M. L. 2002, Science, 295, 93

Barkana, R. \& Loeb, A. 2001, Phys. Rep., 349, 125

Beers, T. C. \& Christlieb, N. 2005, ARAA, 43, 531

Bloom, J. S. 2011, What are Gamma-ray Bursts? (Princeton: Princeton Univ. Press)

Bovill, M. \& Ricotti, M. 2011a, ApJ, 741, 17

Bovill, M. \& Ricotti, M. 2011b, ApJ, 741, 18

Bromm, V., Coppi, P. S., \& Larson, R. B. 2002, ApJ, 564, 23

Bromm, V. \& Larson, R. B. 2004, ARAA, 42, 79

Bromm, V. \& Loeb, A. 2003a, Nature, 425, 812

Bromm, V. \& Loeb, A. 2003b, ApJ, 596, 34

Bromm, V. \& Loeb, A. 2004, New Astron., 9, 353

Bromm, V. \& Loeb, A. 2006, ApJ, 642, 382

Bromm, V., Yoshida, N., Hernquist, L., \& McKee, C. F. 2009, Nature, 459, 49

Bromm, V. \& Yoshida, N. 2011, ARAA, 49, 373

Ciardi, B. \& Ferrara, A. 2005, Space Science Rev., 116, 625

Clark, P. C., Glover, S. C. O., Klessen, R. S., \& Bromm, V. 2011a, ApJ, 727, 110

Clark, P. C., Glover, S. C. O., Smith, R. J., Greif, T. H., Klessen, R. S., \& Bromm, V. 2011b, Science, 331, 1040

Forbes, D. A. \& Kroupa, P. 2011, PASA, 28, 77

Frebel, A. 2010, Astronomische Nachrichten, 331, 474

Frebel, A. \& Bromm, V. 2012, ApJ, 759, 115

Frebel, A., Johnson, J. L., \& Bromm, V. 2007, MNRAS (Letters), 380, L40

Freeman, K. \& Bland-Hawthorn, J. 2002, ARAA, 40, 487

Furlanetto, S. R., Oh, S. P., \& Briggs, F. H. 2006, Phys. Rep., 433, 181

Gardner, J. P., et al. 2006, Space Science Rev., 123, 485

Glover, S. C. O. 2005, Space Science Rev., 117, 445

Greif, T. H., Johnson, J. L., Klessen, R. S., \& Bromm, V. 2008, MNRAS, 387, 1021

Greif, T. H., Glover, S. C. O., Bromm, V., \& Klessen, R. S. 2010, ApJ, 716, 510

Greif, T. H., Springel, V., White, S. D. M., Glover, S. C. O., Clark, P. C., Smith, R. J., Klessen, R. S., \& Bromm, V. 2011, ApJ, 737, 75

Greif, T. H., Bromm, V., Clark, P. C., Glover, S. C. O., Smith, R. J., Klessen, R. S., Yoshida, N., \& Springel, V. 2012, MNRAS, 424, 399

Hosokawa, T., Omukai, K., Yoshida, N., \& Yorke, H. W. 2011, Science, 334, 1250

Hummel, J., Pawlik, A., Milosavljevic, M., \& Bromm, V. 2012, ApJ, 755, 72

Jeon, M., Pawlik, A. H., Greif, T. H., Glover, S. C. O., Bromm, V., Milosavljevic, M., \& Klessen, R. S. 2012, ApJ, 754, 34

Johnson, J. L. \& Bromm, V. 2006, MNRAS, 366, 247

Johnson, J. L. \& Bromm, V. 2007, MNRAS, 374, 1557

Johnson, J. L., Greif, T. H., \& Bromm, V. 2008, MNRAS, 388, 26

Karlsson, T., Bromm, V., \& Bland-Hawthorn, J. 2012, Rev. Mod. Phys., in press (arXiv:1101.4024)

Lamb, D. Q. \& Reichart, D. E. 2000, ApJ, 536, 1

Loeb, A. 2010, How did the first stars and galaxies form? (Princeton: Princeton Univ. Press) 
Maio, U., Ciardi, B., Dolag, K., Tornatore, L., \& Khochfar, S. 2010, MNRAS, 407, 1003

McKee, C. F. \& Ostriker, E. C. 2007, ARAA, 45565

McKee, C. F. \& Tan, J. C. 2008, ApJ, 681, 771

Milosavljević, M., Bromm, V., Couch, S. M., \& Oh, S. P. 2009a, ApJ, 698, 766

Milosavljević, M., Couch, S. M., \& Bromm, V. 2009b, ApJ (Letters), 696, L146

Mirabel, I. F., Dijkstra, M., Laurent, P., Loeb, A., \& Pritchard, J. R. 2011, A\&GA, 528, 149

Oh, S. P. \& Haiman, Z. 2002, ApJ, 569, 558

Pan, T., Kasen, D., \& Loeb, A. 2012, MNRAS, 422, 2701

Pawlik, A., Milosavljević, M., \& Bromm, V. 2011, ApJ, 731, 54

Pawlik, A., Milosavljević, M., \& Bromm, V. 2013, ApJ, 767, 59

Prieto, J., Padoan, P., Jimenez, R., \& Infante, L. 2011, ApJ (Letters), 731, L38

Ricotti, M., Gnedin, N. Y., \& Shull, M. J. 2002a, ApJ, 575, 33

Ricotti, M., Gnedin, N. Y., \& Shull, M. J. 2002b, ApJ, 575, 49

Ritter, J. S., Safranek-Shrader, C., Gnat, O., Milosavljević, M., \& Bromm, V. 2012, ApJ, 761, 56

Safranek-Shrader, C., Agarwal, M., Federrath, C., Dubey, A., Milosavljević, M., \& Bromm, V. 2012, MNRAS, 426, 1159

Salvadori, S. \& Ferrara, A. 2009, MNRAS (Letters), 395, L6

Salvaterra, R., Ferrara, A., \& Dayal, P. 2011, MNRAS, 414, 847

Schneider, R., Omukai, K., Inoue, A. K., \& Ferrara, A. 2006, MNRAS, 369, 1437

Smith, R. J., Hosokawa, T., Omukai, K., Glover, S. C. O., \& Klessen, R. S. 2012 MNRAS, 424, 457

Stacy, A., Greif, T. H., \& Bromm, V. 2010, MNRAS, 403, 45

Stacy, A., Bromm, V., \& Loeb, A. 2011, MNRAS, 413, 543

Stacy, A., Greif, T. H., \& Bromm, V. 2012, MNRAS, 422, 290

Stacy, A., Greif, T. H., Klessen, R. S., Bromm, V., \& Loeb, A. 2013, MNRAS, 431, 1470

Tornatore, L., Ferrara, A., \& Schneider, R. 2007, MNRAS, 382, 945.

Tumlinson, J. 2010, ApJ, 708, 1398

Turk, M. J., Abel, T., \& O'Shea, B. W. 2009, Science, 325, 601

Volonteri, M. 2012, Science, 337, 544

Wang, F. Y., Bromm, V., Greif, T. H., Stacy, A., Dai, Z. G., Loeb, A., \& Cheng, K. S. 2012, ApJ, 760, 27

Willman, B. \& Strader, J. 2012, AJ, 144, 76

Wise, J. H. \& Abel, T. 2007, ApJ, 665, 899

Wise, J. H. \& Abel, T. 2008, ApJ, 685, 40

Wise, J. H., Turk, M. J., Norman, M. L., \& Abel, T. 2012, ApJ, 745, 50

Yoshida, N., Omukai, K., Hernquist, L., \& Abel, T. 2006, ApJ, 652, 6

Yoshida, N., Omukai, K., \& Hernquist, L. 2008, Science, 321, 669 